\title{
Acrosomal integrity and capacitation are not influenced by sperm cryopreservation in the giant panda
}

\author{
R E Spindler, Y Huang ${ }^{1}$, J G Howard, P Wang ${ }^{1}$, H Zhang ${ }^{1}$, G Zhang ${ }^{1}$ and D E Wildt \\ Conservation and Research Center, Smithsonian's National Zoological Park, 1500 Remount Road, Front Royal, \\ Virginia 22630, USA and ${ }^{1}$ China Research and Conservation Center for the Giant Panda, Wolong, China
}

Correspondence should be addressed to R E Spindler; Email: rspindler@torontozoo.ca

\begin{abstract}
Sperm cryopreservation and artificial insemination are important management tools for giant panda breeding and the preservation of extant genetic diversity. This study examined the influence of freeze-thawing on sperm function, specifically capacitation. Sperm from nine giant pandas were assessed before and after rapid ( -40 and $-100{ }^{\circ} \mathrm{C} / \mathrm{min}$ ) cryopreservation by incubation in HEPES-buffered Ham's F10 medium with and without the capacitation accelerators, 3-isobutyl-1-methylxanthine (IBMX) and dibutyryl cyclic AMP (dbcAMP). At 0, 3 and $6 \mathrm{~h}$ of exposure, aliquots were assessed for sperm motility traits and capacitation, defined as the proportion of sperm with intact acrosomes following exposure to solubilised zonae pellucidae (ursid or felid) or calcium ionophore subtracted from the proportion of sperm with intact acrosomes before exposure. Although mean \pm S.E.M. sperm motility post-thaw $(56.1 \pm 3.9 \%$ at $0 \mathrm{~h})$ was less $(P<0.05)$ than pre-freeze $(71.7 \pm 6.0 \%)$, there was no difference $(P>0.05)$ in the proportion of acrosome-intact sperm (fresh, $93.0 \pm 1.7 \%$ versus cryopreservedthawed, $81.7 \pm 4.7 \%$ at $0 \mathrm{~h})$. Incidence of capacitation was greater $(P<0.05)$ in fresh sperm incubated with capacitation accelerators IBMX and dbcAMP $(9 \mathrm{~h}: 50.9 \pm 1.1)$ compared with fresh sperm incubated without accelerators $(9 \mathrm{~h}$ : $41.2 \pm 1.1 \%)$. Frozen-thawed sperm preincubated without accelerators underwent capacitation $(49.6 \pm 1.1 \%)$ to a greater extent $(P<0.05)$ compared with these fresh counterparts. Thawed samples with $(9 \mathrm{~h}: 45.9 \pm 1.4 \%)$ and without accelerators $(9 \mathrm{~h}: 41.2 \pm 1.1 \%)$ did not differ $(P>0.05)$ during the 9-h incubation. We conclude that giant panda spermatozoa (1) undergo capacitation in vitro with or without chemical accelerators and (2) withstand a rapid cryopreservation protocol, including retaining normal acrosomal integrity and functional capacitation ability.

Reproduction (2004) 127 547-556
\end{abstract}

\section{Introduction}

The managed breeding of giant pandas is challenging because males often experience low libido and/or aggressive behaviours toward females (Liu et al. 1998, Zhang et al. 2004). There is a high level of genetic heterozygosity in the ex situ population of $>120$ giant pandas in China (Lu et al. 2001). To avoid potential inbreeding depression in the future, however, it is essential that all the valuable individuals reproduce. Artificial insemination $(\mathrm{Al})$ of fresh semen already plays an important role in breeding giant pandas in captivity, including helping to overcome the problem of sexual incompatibility (Liu 1981, Hodges et al. 1984, Moore et al. 1984). Most Al successes have occurred using fresh spermatozoa (Liu et al. 1979, Moore et al. 1984, Masui et al. 1989, Huang et al. 2001), although a few cubs have been produced by inseminating thawed sperm (Hu \& Wei 1990, Ye et al. 1991, Zhang et al. 1991, Huang et al. 2002).

The genetic management of giant pandas in captivity is recognised as one of the highest priorities for ex situ conservation action in China (Zheng et al. 1997, Yan et al. 2000). The ability to consistently produce offspring by the efficient use of cryopreserved sperm would allow extending sperm viability from days to years and even generations (Wildt et al. 1997). This significantly improves the potential for effective genetic management, and the predicted survival of the species (Harnal et al. 2002). The use of Al with thawed sperm also would facilitate maintaining genetic management through the distribution of genes among geographically dispersed panda populations, including those living in remote breeding centres and zoos. Availability of frozen sperm would permit international transport of genetic material without compromising political and legal restrictions, such as policies established by the United States Fish and Wildlife Service (United-States-Fish-and-Wildlife-Service 1998). Finally, effective sperm cryopreservation could theoretically allow securing robust genetic material of wild giant pandas that are occasionally captured for brief periods. Sperm from these individuals could be considered 'insurance' for the gene pool, with aliquots used periodically to infuse heterozygosity into the ex situ 
population (Wildt et al. 1997). This would eliminate the need to ever extract more giant pandas from native habitats to support zoos.

There are reports on giant panda gamete ultrastructure (Chen et al. 1984), capacitation (Sun et al. 1996), the acrosome reaction (Chen et al. 1989a) and oocyte penetration in vitro (Moore et al. 1984, Chen et al. 1989ab), but all using freshly collected spermatozoa. There are no publications on sperm physiology and function in this species occurring during the freezing/thawing process. Functional viability of a spermatozoon generally depends on sperm (1) progressive motility (Drobnis et al. 1988), (2) acrosome integrity (Suarez et al. 1984, Talbot 1985, Cummins \& Yanagimachi 1986) and (3) the ability to undergo capacitation (Yanagimachi 1994), the acrosome reaction (Yanagimachi 1994) and decondensation (Perreault 1990). Capacitation is the final stage of sperm maturation and is essential for the acrosome reaction and subsequent zona pellucida (ZP) penetration and oocyte fertilisation (Yanagimachi 1994).

The present study was one of a series of investigations to assess the influence of seminal processing and cryopreservation/thawing on giant panda sperm function. Here, we focused on the phenomenon of sperm capacitation in vitro. Spermatozoa of some species undergo capacitation spontaneously in vitro, whereas others require accelerators such as 3-isobutyl-1-methylxanthine (IBMX) (Visconti et al. 1999, Dorval et al. 2002, Thundathil et al. 2002) or dibutyryl cyclic AMP (dbcAMP) (Leclerc et al. 1996, Visconti et al. 1999, Lefievre et al. 2000). Our study was designed to determine the dynamics of giant panda sperm capacitation in the presence and absence of IBMX and dbcAMP. Cryopreservation is also known to impede sperm function via damaged acrosomal membranes (RodriguezMartinez et al. 1997, Burgess et al. 2001). Thus, our second objective was to determine if freeze-thawing induced membrane damage which, in turn, disrupted the ability of giant panda sperm to acrosome react.

In mammalian species studied to date, the acrosome reaction is generally readily induced in capacitated sperm by co-culture with conspecific (Uto et al. 1988, Yoshimatsu et al. 1988, Pukazhenthi et al. 1996) or heterospecific (Lee et al. 1987, Yoshimatsu et al. 1988, Slavik et al. 1990) ZP. Due to the lack of giant panda oocytes (and thus ZP), we tested the efficacy of solubilised ZP from felid and ursid oocytes, i.e. other carnivores. These findings were compared with the effectiveness of standard chemical induction (calcium ionophore A283187; Cl) commonly used in other taxa including the bovid (Byrd 1981, Parrish et al. 1988, 1989), the cervid (Harnal et al. 2001) and the felid (Long et al. 1996, Pukazhenthi et al. 1998). Overall, our approach here was to begin to understand capacitation and the physiological and biophysical consequences of processing and cryopreserving giant panda spermatozoa.

\section{Materials and Methods}

\section{Animals and source of ZP for capacitation assessments}

One ejaculate was collected from each of nine giant pandas (5-16 years of age; weighing $79.5-130.0 \mathrm{~kg}$ each) maintained at the China Research and Conservation Center for the Giant Panda in the Wolong Nature Reserve. Males were housed singly in (1) naturalistic enclosures of approximately $300 \mathrm{~m}^{2}$ in size and connected to indoor areas of $8 \mathrm{~m}^{2}$, with olfactory and auditory contact with females in adjacent enclosures or (2) concrete and grass enclosures of approximately $35 \mathrm{~m}^{2}$ and connected to indoor areas of $8 \mathrm{~m}^{2}$, with olfactory, auditory and visual contact with neighbouring females. Males were moved between enclosures of the same or alternate type at irregular intervals, but rarely went more than 1-2 weeks without being in an enclosure immediately adjacent to an adult female. Fresh bamboo ( $\sim 20$ stalks) was provided up to seven times daily and supplemented with high protein concentrate consisting of soybean, bamboo powder, corn, rice and vitamin/mineral additives ( $M$ Edwards, personal communication). Water was available ad libitum. All semen samples were collected during the spring (February-May) breeding season (Schaller et al. 1985).

ZP for functionality assays were collected from oocytes recovered from two species, the domestic cat (Felis catus) and the black bear (Ursus americanus). The former were collected from ovaries provided by local veterinary clinics in the USA conducting ovariohysterectomies in companion cats (Johnston et al. 1989, Wolfe \& Wildt 1996). The oocytes underwent in vitro maturation (Johnston et al. 1989, Wolfe \& Wildt 1996), zonae were then separated from cytoplasm by continued pipetting with undersized (slightly smaller than the width of the oocyte) pipettes to rupture the zonae. Zonae were then washed three times in $2 \mathrm{ml} \mathrm{Ham}$ 's $\mathrm{F} 10$ medium plus $5 \%$ fetal calf serum and $25 \mathrm{mmol} / \mathrm{I}$ HEPES (HF10) before being placed in a cryovial tube (Nunc, Rochester, NY, USA) with HF10 to a concentration of four zonae/ $\mu$. These cryovial tubes were then placed in a sonicating bath (Precision Scientific, Winchester, VA, USA) until no solid zona remained (average $45 \mathrm{~min}$ ). This solution was then divided into $25 \mu \mathrm{l}$ aliquots and frozen until needed for sperm co-incubation. Ursid oocytes were recovered from freeze-thawed ovarian tissue made available through co-operation with hunters and biologists in the USA and Canada during seasonal culls of local, wild black bears. These ovaries were excised between 2 and $12 \mathrm{~h}$ after death, frozen on dry ice and then later transported to the laboratory where the ovaries were thawed and minced (as described previously) (Johnston et al. 1994) to collect oocytes. Ursid zonae were prepared as for felid zonae, with the exception that ursid oocytes did not undergo in vitro maturation. It is likely that the cytoplasm of these oocytes was no longer functional as a result of ovary handling before oocyte recovery. The number of ursid oocytes available was 
much lower than that of felid oocytes, limiting the number of time-points that sperm could be exposed to this treatment. Thus, the 6-h time-point was chosen to compare the effects of ursid with felid zonae on giant panda sperm because it was assumed that this would be the time of maximum capacitation and sperm viability and, therefore, offer the greatest sensitivity. All procedures used in this study were submitted to and approved by the Smithsonian's National Zoological Park's Institutional Animal Care and Use Committee.

\section{Semen collection/analysis}

Semen was collected by electroejaculation after each male was induced into a surgical plane of anaesthesia by an intramuscular injection of $10 \mathrm{mg} / \mathrm{kg}$ ketamine $\mathrm{HCl}$ (Ketaset; Fort Dodge Laboratories Inc., Fort Dodge, IA, USA). Deep anaesthesia was maintained with $0-5 \%$ isofluorane gas, as needed. The electroejaculation technique has been described (Platz et al. 1983, Howard 1993, 1999, Spindler et al. 2001) and briefly relied on using a $2.6 \mathrm{~cm}$ diameter rectal probe with three longitudinal, stainless-steel electrodes and a $60 \mathrm{~Hz}$ sine wave stimulator (P.T. Electronics, Boring, OR, USA). A standardised set of low voltage stimulations $(2-8 \mathrm{~V})$ over three series of 20-30 stimuli each was adequate to elicit ejaculation. The entire semen collection interval generally required $<20 \mathrm{~min}$.

Penile erection occurred during stimulation, and semen was collected into a sterile glass container. Seminal volume was immediately recorded by pipetting, and $\mathrm{pH}$ was measured using $<5 \mu \mathrm{l}$ semen on Color pHast indicator strips (EM Science, Gibbstown, NJ, USA). A seminal $\mathrm{pH}$ value in the normally basic range (7.5-9.0) indicated that urine contamination had not occurred. An additional $10 \mu \mathrm{l}$ sample from each electroejaculation series was used to determine sperm concentration using a haemocytometer (Howard et al. 1990). A $5 \mu \mathrm{l}$ seminal aliquot was examined at $200 \times$ (Olympus BX40 microscope) for a subjective estimate of sperm motility traits, including percent sperm motility and progressive motility (i.e. forward progression on a $0-5$ scale, with 5 indicative of rapid forward sperm movement and 0 indicating no forward progression (Howard 1993). Within each ejaculation series, samples differing from each other by $<10 \%$ in sperm motility and progressive status were combined. Spermatozoa were maintained at $37^{\circ} \mathrm{C}$ throughout assessment and processing using a water bath or dry-bath incubator (Fisher, Hanover Park, IL, USA).

Unless otherwise stated, all chemicals were purchased from Sigma Chemical Co. (St Louis, MO, USA). A $5 \mu \mathrm{l}$ aliquot of the combined electroejaculate was removed and added to $100 \mu \mathrm{l}$ fixative $(0.3 \%$ glutaraldehyde in phosphate-buffered serum) for morphologic examination of spermatozoa (200/sample) by phase-contrast microscopy ( $\times 630)$ (Howard 1993). Spermatozoa were categorised as normal or as having one of the following anomalies, including an abnormal head (i.e. macrocephaly, (a)

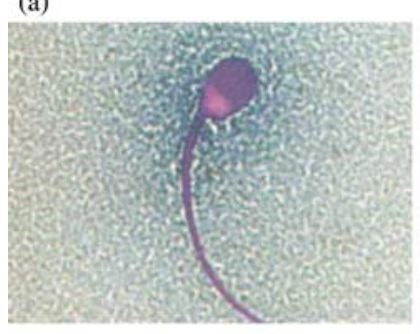

(c)

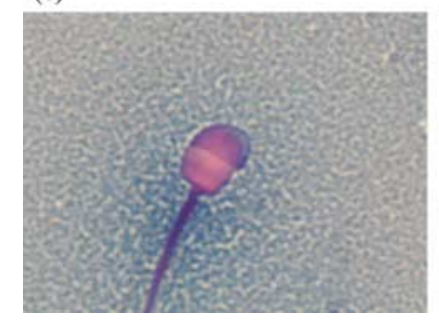

(b)

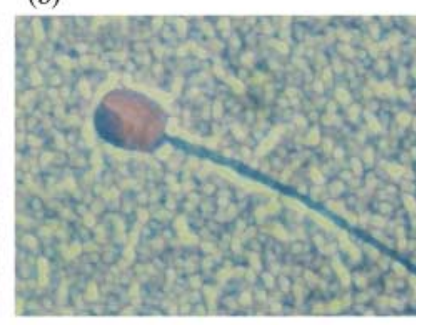

(d)

Figure 1 Acrosome intact sperm appear blue (a) whereas damaged acrosomes are evident by mottled or patchy staining (b) or a detached membrane (c). Sperm with a missing acrosome have a white or pink cap, with no blue staining evident (d).

microcephaly and bicephaly), abnormal acrosome, abnormal midpiece, coiled flagellum, bent midpiece with cytoplasmic droplet, bent midpiece without cytoplasmic droplet, bent flagellum with cytoplasmic droplet, bent flagellum without cytoplasmic droplet, proximal cytoplasmic droplet and distal cytoplasmic droplet (Howard 1993). The proportion of spermatozoa with abaxial attachment of the midpiece was noted but not considered an abnormality (Moore et al. 1984). Acrosomal integrity was evaluated using the rose bengal/fast green stain (Pope et al. 1991). Spermatozoa were stained for $90 \mathrm{~s}$, smeared on a glass slide, allowed to air-dry, and a minimum of 100 spermatozoa were examined by light microscopy $(\times 630)$. Cells then were categorised as having: (1) an intact acrosome whereby the acrosomal membrane stained blue and remained in contact with the sperm head (Fig. 1a); (2) a damaged acrosome whereby the membrane was damaged (Fig. 1b), or was separate from the sperm head (Fig. 1c); or (3) a missing acrosome (Fig. 1d).

\section{Capacitation assessment}

After combining series into a single ejaculate per male, a $0.5 \mathrm{ml}$ aliquot was removed immediately, subdivided into two and diluted with (1) HF10 or (2) HF10 containing an additional supplement of $200 \mu \mathrm{mol} / \mathrm{I}$ IBMX and $1 \mathrm{mmol} / \mathrm{I}$ dbcAMP (CHF10). These diluted aliquots were centrifuged (200 $\mathrm{g} 8 \mathrm{~min}$ ) and the supernatants discarded before each pellet was resuspended in either HF10 or CHF10 (keeping medium consistent before and after centrifugation). Spermatozoa were resuspended in $0.5 \mathrm{ml}$ of either HF10 or CHF10 at a final concentration of $50 \times 10^{6}$ cells $/ \mathrm{ml}$ and incubated at $37^{\circ} \mathrm{C}$ for $6 \mathrm{~h}$.

At 0,3 and $6 \mathrm{~h}$ after resuspension, $5 \mu \mathrm{l}$ samples were removed and assessed for motility and forward progressive 
status. Additional $10 \mu \mathrm{l}$ aliquots were removed and diluted with an equal volume of one of four treatments: (1) HF10 only (control for ZP); (2) HF10 containing solubilised cat (all time-points) or bear (6-h time-point only) ZP to a final concentration of 2 zonae/ $\mu \mathrm{l}$; (3) HF10 containing $5 \%$ dimethylsulfoxide (as a control for Cl); or (4) HF10 containing $1 \mu \mathrm{mol} / \mathrm{l} \mathrm{Cl}$. Each of the four aliquot/treatment suspensions was incubated under mineral oil for $30 \mathrm{~min}$ at $37^{\circ} \mathrm{C}$. At the end of the 30-min incubation, acrosomal integrity was assessed (as above). Percentage of capacitated spermatozoa was defined as the proportion of acrosome intact sperm in treatments no. 1 and no. 3 (controls) minus the percentage of sperm with intact acrosomes in treatments no. 2 and no. 4.

\section{Sperm cryopreservation and thawing}

The remainder of the ejaculate not used for the capacitation study was diluted immediately (within 15 min of original collection) with commercially available TEST egg yolk buffer (Irvine Scientific, Santa Ana, CA, USA) modified to contain $5 \%$ glycerol. Semen was diluted to give a final concentration of $400 \times 10^{6}$ motile sperm $/ \mathrm{ml}$ in $15 \mathrm{ml}$ conical, plastic tubes (Falcon, Bedford, MA, USA) and then placed in a water jacket $\left(400 \mathrm{ml} 37^{\circ} \mathrm{C}\right.$ water) before being placed in a refrigerator to cool slowly to $4{ }^{\circ} \mathrm{C}$ over $4 \mathrm{~h}$, as verified by a thermocouple (Brandt, Prairieville, LA, USA). Cooled semen was pipetted into $0.25 \mathrm{ml}$ sterile, plastic straws (Veterinary Concepts, Spring Valley, WI, USA) and sealed using a Nyclave impulse heat sealer (The Lorvic Corporation, St Louis, MO, USA). Straws were placed $7.5 \mathrm{~cm}$ over liquid nitrogen (LN) for $1 \mathrm{~min}$ and then $2.5 \mathrm{~cm}$ above $\mathrm{LN}$ for $1 \mathrm{~min}$ to achieve a rapid cryopreservation rate of $-40{ }^{\circ} \mathrm{C} / \mathrm{min}$ and $-100^{\circ} \mathrm{C} / \mathrm{min}$ respectively (as determined by previous control thermocouple testing). Frozen samples were plunged into the liquid phase of LN and stored for at least $24 \mathrm{~h}$ before thawing. Spermatozoa were thawed by exposing the straw to air for $10 \mathrm{~s}$, followed by plunging the straw into a $37^{\circ} \mathrm{C}$ waterbath for $30 \mathrm{~s}$. Both ends of the straw were cut off and the thawed sample allowed to flow into a $5 \mathrm{ml}$ sterile plastic tube (Falcon) where it was diluted immediately in $2 \mathrm{ml} \mathrm{HF10} \mathrm{at}$ $37^{\circ} \mathrm{C}$. The thawed sample was split into two equal aliquots, diluted in HF10 and each centrifuged simultaneously in a $1.5 \mathrm{ml}$ Eppendorf microcentrifuge tube (200 $\mathrm{g} 8 \mathrm{~min})$. The supernatant was discarded, and one sperm pellet was resuspended in HF10, whereas the other was suspended in CHF10 (both at a final concentration of $50 \times 10^{6} \mathrm{cell} / \mathrm{ml}$ in $0.5 \mathrm{ml}$ ) at $37^{\circ} \mathrm{C}$. Thawed spermatozoa then were assessed for capacitation exactly as described above for fresh counterparts.

\section{Statistical analysis}

Differences among treatments were evaluated by ANOVA and Dunnett's multiple comparison testing (Miller 1981). Percentage data were arcsine transformed before analysis.
Data are expressed as means \pm S.E.M. Correlation coefficients among sperm traits were determined using the Pearson-product moment correlation curve (Box et al. 1978).

\section{Results}

Seminal volume $(2.8 \pm 0.4 \mathrm{ml})$ and sperm concentration $\left(1.1 \pm 0.3 \times 10^{9} \mathrm{sperm} / \mathrm{ml}\right)$, initial motility $(76.1 \pm 4.4 \%)$, forward progression rating $(3.4 \pm 0.2)$ and acrosomal integrity $(93.6 \pm 1.7 \%$ intact) for giant panda ejaculates collected here were consistent with previous reports (Platz et al. 1983, Chen et al. 1994). Most spermatozoa were morphologically normal $(84.2 \pm 3.0 \%)$, although many $(74.9 \pm 8.2 \%)$ displayed the interesting abaxial attachment trait for the head/midpiece region as noted previously (Moore et al. 1984). There is no evidence that this is an abnormal morphotype for the giant panda spermatozoon; thus, these sperm were categorised as normal if other abnormalities were absent. Furthermore, there were no differences $(P>0.05)$ among males in any of the ejaculate traits measured (data not shown). In short, all subject males produced prodigious amounts of motile, high quality spermatozoa.

The motility of fresh (control) sperm samples declined over time (Fig. 2) to about $20 \%$ after a 9 -h incubation in HF10. The gradual decline in sperm motility was not accelerated or otherwise altered $(P>0.05)$ by the presence of IBMX and dbcAMP in the medium. For example, whereas overall sperm motility in $\mathrm{HF} 10$ at $9 \mathrm{~h}$ was $27.0 \pm 5.6 \%$, this same trait was similar $(P>0.05)$ in CHF10 containing the combined IBMX and dbcAMP $(13.3 \pm 5.4 \%)$. There also was a loss $(P<0.05)$ in sperm motility immediately after thawing $(56.1 \pm 3.9 \%$ motile $)$ compared with pre-freeze motility (71.7 $\pm 6.0 \%$; Fig. 2$)$. Nonetheless, subsequent sperm motility values for fresh and frozen-thawed aliquots were comparable within time-points $(P>0.05)$ (Fig. 2). Overall motility in thawed samples did not $(P>0.05)$ decline more rapidly than

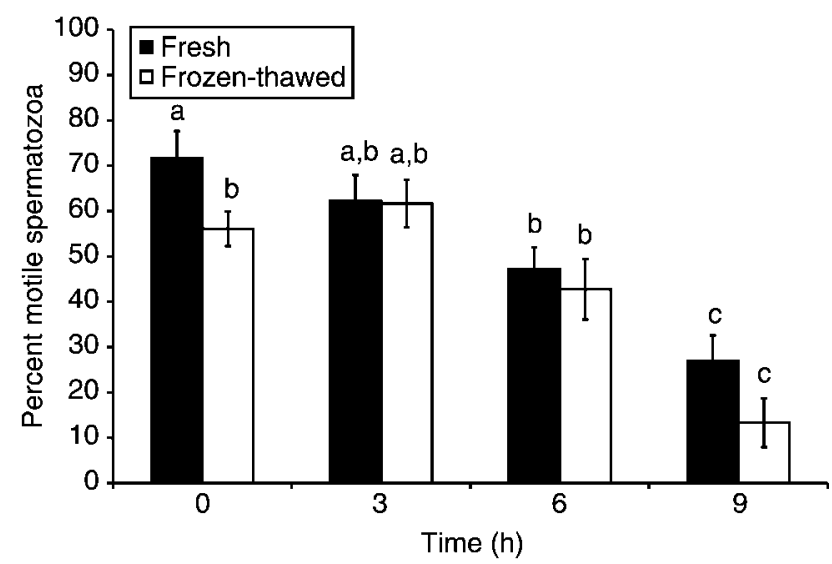

Figure 2 Motility of giant panda spermatozoa incubated in vitro in HF10. Different superscripts within and across time-periods differ $(P<0.05)$. 
fresh counterparts (the gradient of decline in motility was $-4.91 \% / \mathrm{h}$ for frozen-thawed versus $-4.97 \% / \mathrm{h}$ for fresh aliquots). A similar observation was made for progressive motility ratings in that no differences $(P>0.05)$ were measured between the same time-periods for fresh $(0 \mathrm{~h}$, $3.4 \pm 0.2 ; 3 \mathrm{~h}, 3.4 \pm 0.2 ; 6 \mathrm{~h}, 3.1 \pm 0.2 ; 9 \mathrm{~h}, 1.3 \pm 0.6)$ versus thawed $(0 \mathrm{~h}, 3.1 \pm 0.3 ; 3 \mathrm{~h}, 3.3 \pm 0.2 ; 6 \mathrm{~h}$, $2.8 \pm 0.2 ; 9 \mathrm{~h}, 1.1 \pm 0.4)$ aliquots.

There was a comparable $(P>0.05)$ percentage of fresh $(93.0 \pm 1.7 \%)$ and thawed $(81.7 \pm 4.7 \%)$ spermatozoa with intact acrosomes at $0 \mathrm{~h}$. The proportion of fresh spermatozoa with intact acrosomes decreased $(P<0.05)$, albeit modestly $(\sim 10 \%)$ within $3 \mathrm{~h}$ of incubation and regardless of medium (HF10 or CHF10) (Fig. 3). There was a similar trend in acrosomal integrity for thawed sperm with an overall decline $(P>0.05)$ of $\sim 6 \%$ from 0 to $3 \mathrm{~h}$ of culture. Within time-period and medium, there were no differences $(P>0.05)$ in the proportions of fresh versus thawed sperm with intact acrosomes (Fig. 3). However, acrosomal integrity degraded $(P<0.05)$ more severely in cells that were both cryopreserved and incubated in CHF10 for 6 and $9 \mathrm{~h}$ compared with fresh sperm incubated without capacitation accelerators (Fig. 3).

The number of spermatozoa with intact acrosomes decreased markedly $(P<0.05)$ in response to the addition of heterologous cat or bear ZP to the culture medium compared with spermatozoa incubated in control medium (indicating acrosome reaction and therefore successful capacitation) (Fig. 4). For example, because $87 \%$ of spermatozoa from male 4 incubated in control HF10 had intact acrosomes in the control treatment and only $30 \%$ of cat ZP-exposed spermatozoa had intact acrosomes at $6 \mathrm{~h}$, then $57 \%$ of spermatozoa at this time-period in HF10 were considered capacitated.
Compared with $0 \mathrm{~h}$, percentage of capacitated spermatozoa was greater $(P<0.05)$ in all treatments after $3 \mathrm{~h}$ and increased $(P<0.05)$ further by $6 \mathrm{~h}$ (Fig. 5). No further increase $(P>0.05)$ was observed at $9 \mathrm{~h}$. Fresh sperm incubated in CHF10 had a greater $(P<0.05)$ incidence of capacitation than HF10-incubated sperm at 3, 6 and $9 \mathrm{~h}$ (Fig. 5). Fewer frozen-thawed CHF10-incubated sperm completed capacitation by $6 \mathrm{~h}$ compared with fresh counterparts. On the contrary, more frozen-thawed sperm had undergone capacitation after a 9-h incubation in HF10 compared with fresh counterparts (Fig. 5). The extent and rate of capacitation of thawed sperm was not otherwise different $(P>0.05)$ compared with fresh counterparts. Percentage of capacitated spermatozoa immediately post-thaw $(0 \mathrm{~h})$ was $<5 \%$ in all treatments, but increased $(P<0.05)$ to $27.7 \pm 1.2 \%$ and $32.1 \pm 1.5 \%$ after a 3 -h incubation in HF10 and CHF10 respectively (Fig. 5). After a 6-h incubation, an even greater number $(P<0.05)$ of thawed sperm incubated in HF10 (43.3 $\pm 1.1 \%)$ and CHF10 (40.4 $\pm 1.4 \%)$ were capacitated. An additional 3-h incubation resulted in more capacitated sperm only in the frozen-thawed HF10incubated sperm. The presence of IBMX and dbcAMP in the medium had no impact $(P>0.05)$ on the overall incidence or rate at achieving sperm capacitation in thawed sperm aliquots (Fig. 5).

The bear ZP emulsion was less effective $(P>0.05)$ at inducing the acrosome reaction (by $6 \mathrm{~h}$ ) in fresh (HF10, $26.0 \pm 2.8 \%$ ) and thawed sperm (HF10, $21.7 \pm 5.2 \%$ ) compared with either cat ZP (fresh sperm, HF10, $6 \mathrm{~h}$ : $43.6 \pm 1.1 \%$; thawed, HF10, $6 \mathrm{~h}: 43.3 \pm 1.1 \%$ ) or $\mathrm{Cl}$ (fresh sperm, HF10, $6 \mathrm{~h}: 42.1 \pm 1.8 \%$; thawed, HF10, $6 \mathrm{~h}$ : $41.4 \pm 2.1 \%$ ). Sperm categorised as not intact could be either damaged or missing (see Fig. 1). A significant

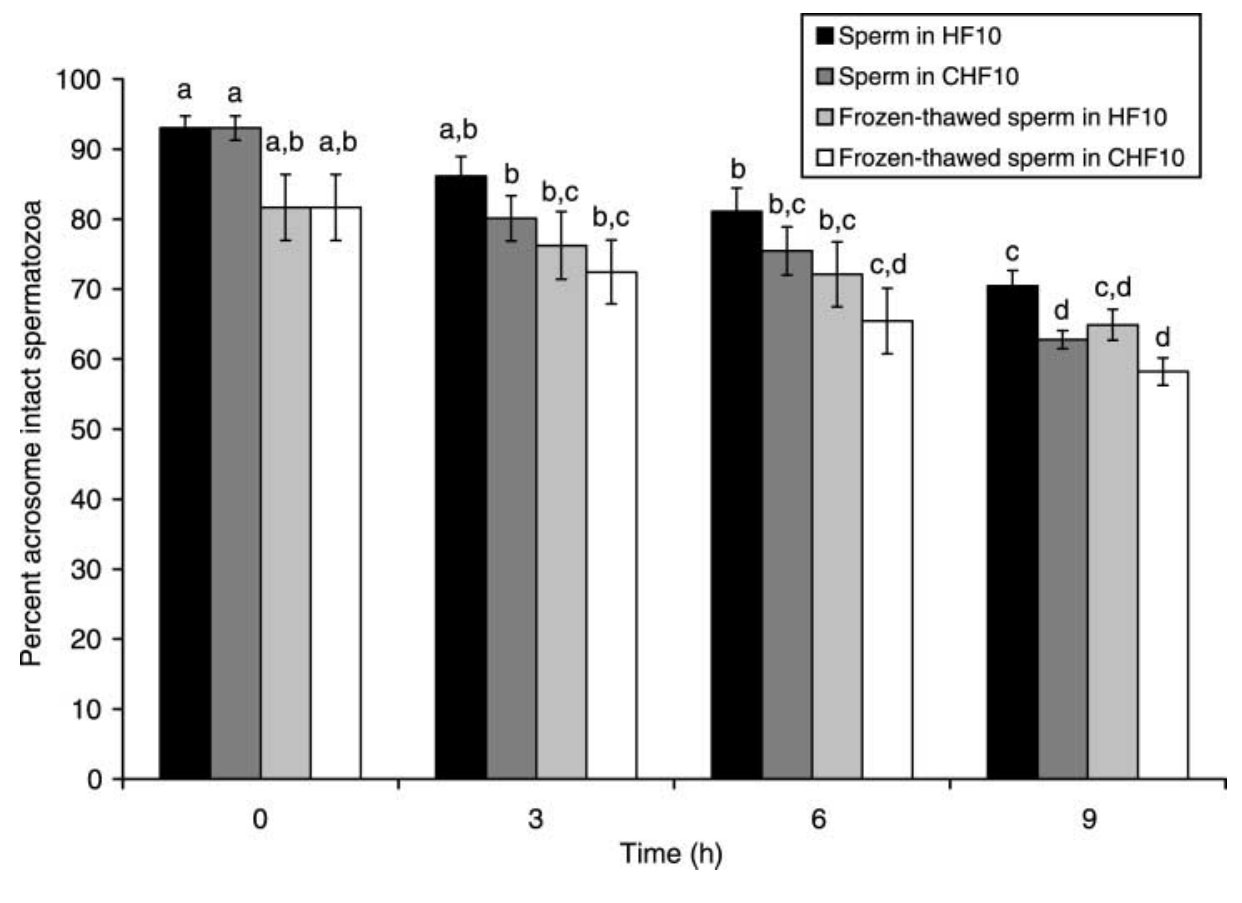

Figure 3 Acrosomal integrity of fresh giant panda spermatozoa incubated in HF10 and CHF10 and frozen-thawed spermatozoa incubated in HF10 and CHF10. Different superscripts within and between time-periods differ $(P<0.05)$. 


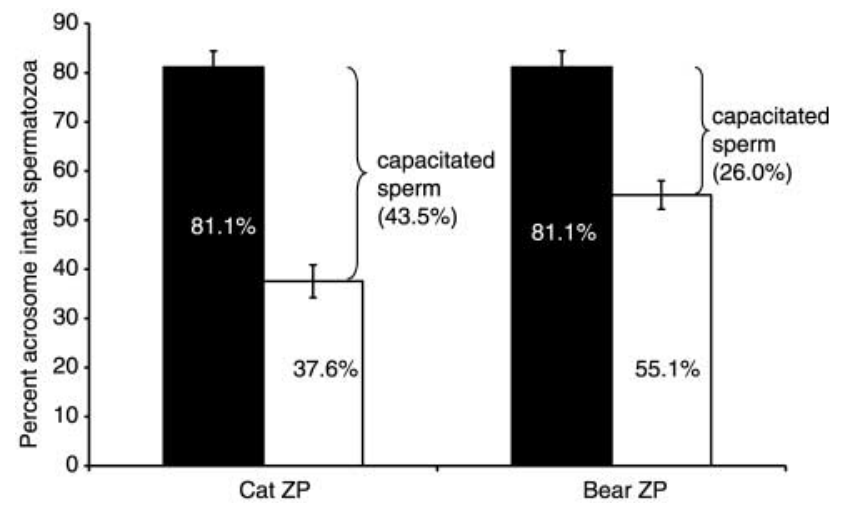

Figure 4 Acrosome-intact fresh giant panda spermatozoa after incubation in HF10 control (solid bars) versus solubilised cat or bear ZP (open bars) at $6 \mathrm{~h}$. The difference between these treatments is the percentage of capacitated sperm.

proportion of sperm categorised as having damaged acrosomes in control sperm increased from $5.3 \pm 2.1 \%$ (HF10) at $0 \mathrm{~h}$ to $14.3 \pm 1.9 \%$ at $9 \mathrm{~h}$. There was a higher proportion of damaged sperm in HF10 at $0 \mathrm{~h}$ following cryopreservation (HF10: $13.1 \pm 2.1 \%$ ), but at $3 \mathrm{~h}$ this difference was no longer evident (fresh: $9.4 \pm 1.7 \%$ versus thawed: $16.4 \pm 2.6 \%$ ). Fresh and frozen sperm sustained this damage regardless of whether or not incubation medium contained capacitation accelerators. However, there was a trend $(P>0.05)$ toward greater damage to frozen-thawed sperm after incubation with capacitation accelerators for $9 \mathrm{~h}(25.5 \pm 0.8 \%)$ compared with thawed sperm incubated without accelerators (20.2 $\pm 1.4 \%)$. The number of sperm assessed to be capacitated after incubation with cat ZP was less $(P>0.05)$ than when $\mathrm{Cl}$ was used to induce the acrosome reaction at $9 \mathrm{~h}$ (Fig. 6). There was no difference in the ability of $\mathrm{Cl}$ and $\mathrm{ZP}$ to induce the acrosome reaction at any other time-point (Fig. 6).
Damage to fresh and thawed sperm over time was not different $(P>0.05)$ between sperm exposed to cat ZP versus $\mathrm{Cl}$ (Table 1).

\section{Discussion}

Giant panda spermatozoa were capable of capacitating in vitro over a 6-h interval of incubation with or without the use of the accelerators IBMX and dbcAMP. Although there was a modest loss of cellular motility post-thaw, the methods described here allowed freeze-thawing of giant panda sperm without compromising acrosomal integrity. Both ursid and felid zonae emulsions elicited the acrosome reaction in giant panda spermatozoa, supporting data indicating that the triggers to this phenomenon are not species specific (Yanagimachi 1994). Most importantly, the methods utilised here for freeze-thawing these cells did not compromise the ability of giant panda sperm to undergo capacitation.

Given the importance of sperm motility (Drobnis et al. 1988) and acrosome integrity (Suarez et al. 1984, Talbot 1985, Cummins \& Yanagimachi 1986) to successful fertilisation, the discovery of a high proportion of motile, acrosome-intact sperm after cryopreservation was encouraging, but perhaps still not sufficient for normal fertilisation. For example, the incidence of damaged acrosomes found in thawed, CHF10-incubated and even control sperm over time may have indicated that these cells were becoming damaged due to contact with other sperm or the plastic incubation tube. It is possible that damage or premature 'capacitation-like' changes were occurring to membranes spontaneously, during freezethawing, or exposure to medium containing the accelerators (Gillan et al. 1997, Gillan \& Maxwell 1999, Green \& Watson 2001, Samper 2001). Alternatively, these sperm labelled damaged may have actually been partially

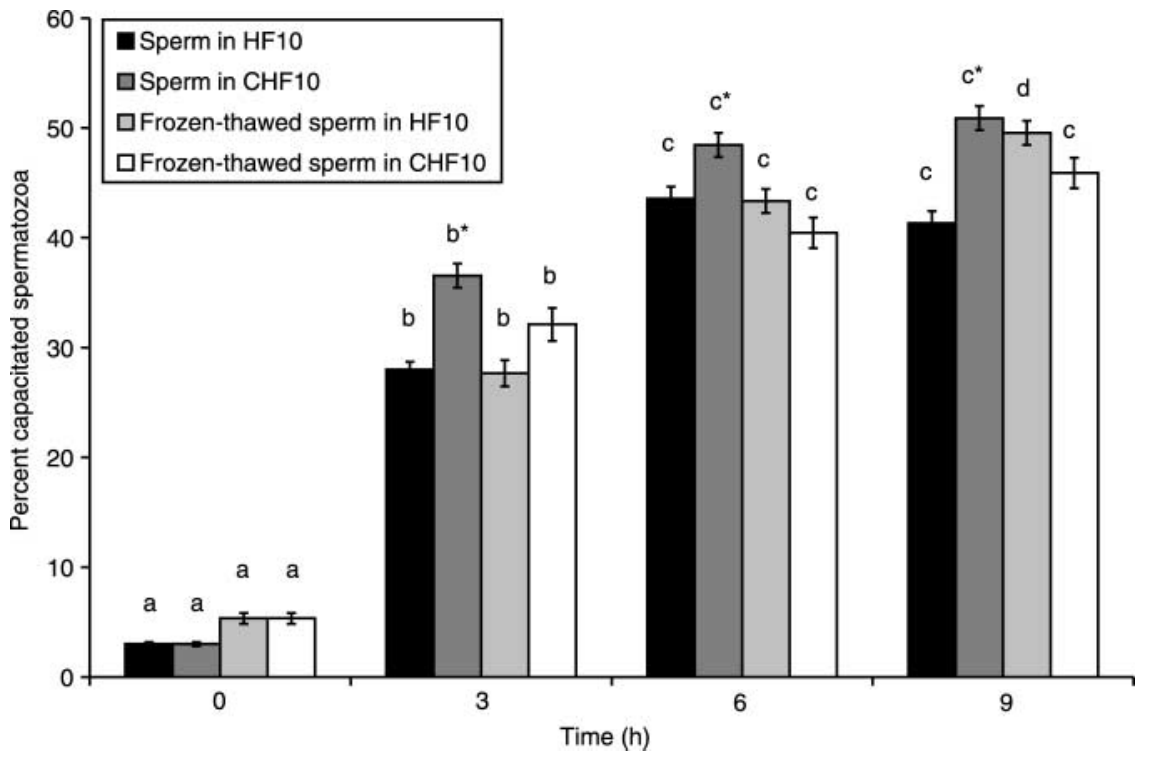

Figure 5 Percent capacitated fresh and thawed giant panda spermatozoa after incubation in HF10 and CHF10 as determined by incubation with cat ZP. Within each treatment, different superscripts differ over time $(P<0.05)$. *Within the time-period, the value is different $(P<0.05)$ from the HF10 counterpart. 


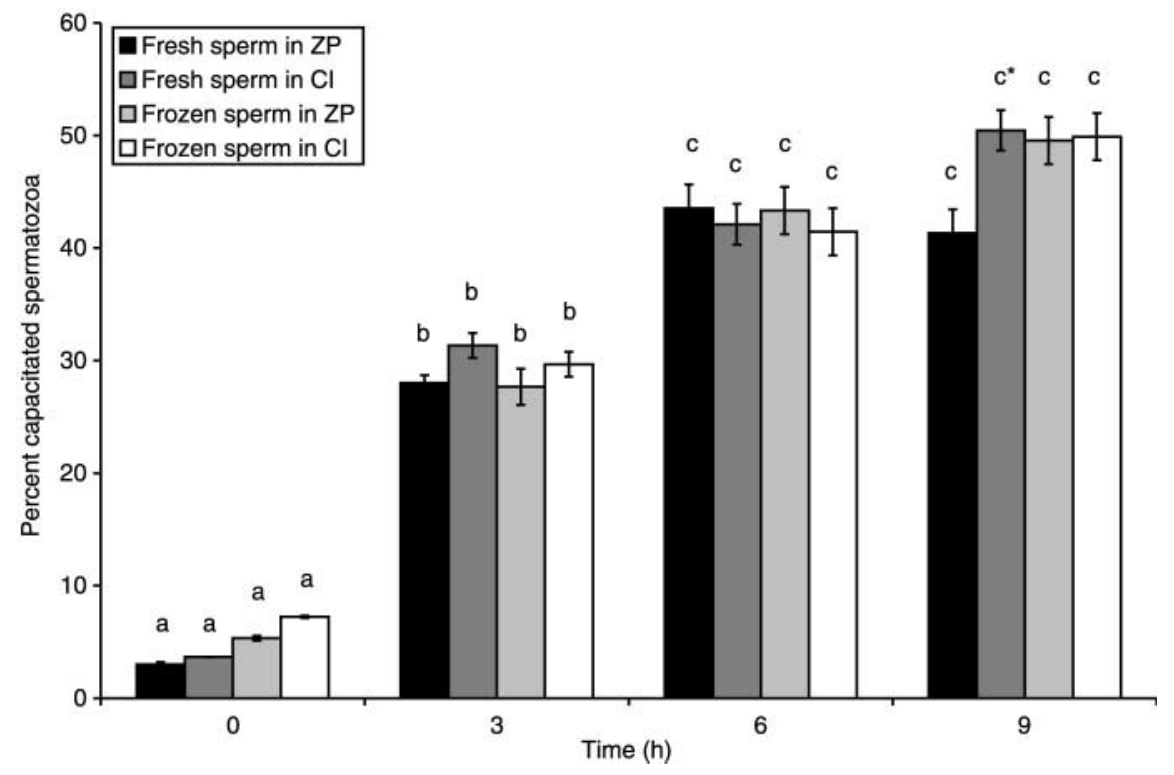

Figure 6 Estimated capacitation of fresh and cryopreserved spermatozoa as determined by incubation with solubilised cat $Z \mathrm{P}$ versus $\mathrm{Cl}$. Within each treatment, different superscripts differ over time $(P<0.05)$. *Within the time-period, the value is different $(P<0.05)$ from the ZP counterpart. capacitated sperm as has been found in felids (Long et al. 1996). This appears to have been supported by the finding of negligible damage early in incubation, followed by an increase over time, paralleling the observed incidence of capacitation. In either case, an acrosome that reacts or that is damaged before interacting with the oocyte will likely result in a non-functional spermatozoon (Kopf \& Gerton 1991). However, the percentage of intact acrosomes does not always correlate positively with embryo development after insemination in vitro ( $\mathrm{O}^{\prime}$ Brien \& Roth 2000). Further, fertility may be unaffected, as shown in a recent study of sheep where pregnancy success was similar using inseminates containing a high versus a low percentage of acrosome-intact sperm, if adjustments were made to ensure that an equal number of intact sperm were inseminated (Gil et al. 2002).

Over our 9-h incubation, up to one-half of the sperm in each sample were intact and capacitated (Fig. 4). It is not known if this time-line is consistent with the course of events in vivo; however, the rate and extent of capacitation

Table 1 Percentage of fresh and thawed giant panda spermatozoa categorised as having missing and damaged acrosomes after exposure to cat ZP or Cl. Values are means \pm S.E.M.

\begin{tabular}{lrrrrr}
\hline & \multicolumn{2}{c}{ Fresh aliquots } & & \multicolumn{2}{c}{ Thawed aliquots } \\
\cline { 2 - 3 } \cline { 5 - 6 } \cline { 5 - 6 } & Missing & Damaged & & Missing & Damaged \\
\hline $0 \mathrm{~h}, \mathrm{ZP}$ & $3.0 \pm 0.4^{\mathrm{a}}$ & $7.0 \pm 2.2^{\mathrm{a}}$ & & $6.0 \pm 2.0^{\mathrm{a}}$ & $17.7 \pm 2.8^{\mathrm{a}}$ \\
$0 \mathrm{~h}, \mathrm{Cl}$ & $2.5 \pm 0.9^{\mathrm{a}}$ & $9.4 \pm 2.1^{\mathrm{a}}$ & & $5.6 \pm 3.2^{\mathrm{a}}$ & $20.4 \pm 3.1^{\mathrm{a}}$ \\
$3 \mathrm{~h}, \mathrm{ZP}$ & $18.1 \pm 4.2^{\mathrm{b}}$ & $24.8 \pm 3.2^{\mathrm{b}}$ & & $26.2 \pm 4.7^{\mathrm{b}}$ & $25.2 \pm 3.3^{\mathrm{a}, \mathrm{b}}$ \\
$3 \mathrm{~h}, \mathrm{Cl}$ & $20.7 \pm 3.6^{\mathrm{b}}$ & $28.1 \pm 3.1^{\mathrm{b}}$ & & $23.8 \pm 4.2^{\mathrm{b}}$ & $33.6 \pm 5.9^{\mathrm{b}}$ \\
$6 \mathrm{~h}, \mathrm{ZP}$ & $40.3 \pm 7.4^{\mathrm{c}}$ & $22.1 \pm 2.3^{\mathrm{b}}$ & & $43.4 \pm 3.7^{\mathrm{c}}$ & $27.8 \pm 4.7^{\mathrm{a}, \mathrm{b}}$ \\
$6 \mathrm{~h}, \mathrm{Cl}$ & $43.7 \pm 8.4^{\mathrm{c}}$ & $25.2 \pm 3.6^{\mathrm{b}}$ & & $43.4 \pm 7.1^{\mathrm{c}}$ & $30.2 \pm 5.2^{\mathrm{a}, \mathrm{b}}$ \\
$9 \mathrm{~h}, \mathrm{ZP}$ & $52.0 \pm 4.6^{\mathrm{c}}$ & $18.9 \pm 2.2^{\mathrm{b}}$ & & $62.4 \pm 4.9^{\mathrm{c}}$ & $22.2 \pm 5.3^{\mathrm{a}, \mathrm{b}}$ \\
$9 \mathrm{~h}, \mathrm{Cl}$ & $63.0 \pm 2.8^{\mathrm{d}}$ & $23.4 \pm 3.1^{\mathrm{b}}$ & & $61.6 \pm 5.7^{\mathrm{c}}$ & $25.4 \pm 5.9^{\mathrm{a}, \mathrm{b}}$ \\
\hline
\end{tabular}

Different superscripts within the same column differ significantly $(P<0.05)$. were similar to that reported for bovine (Byrd 1981, Parrish et al. 1988), porcine (Hunter \& Hall 1974), feline (Andrews et al. 1992) and human (Lee et al. 1987) spermatozoa. Previous studies have performed ultrastructural and chemical studies of giant panda sperm capacitation (Chen et al. 1989b Sun et al. 1996), but a detailed report of the expected time-course for capacitation has not been performed before. However, our results concur with those of Sun et al. (1996) who report that at least some giant panda sperm undergo capacitation by $5.5 \mathrm{~h}$ and $6.5 \mathrm{~h}$ of incubation.

It is somewhat surprising that $\mathrm{Cl}$ and zonae emulsions were almost equally capable of eliciting the acrosome reaction, as a previous report has found that $\mathrm{Cl}$ is able to induce capacitation in up to $40 \%$ more sperm than solubilised zona (Long et al. 1996). However, this may be accounted for by the fact that the giant panda sperm studied here were exposed to twice the concentration of emulsified zonae and one-quarter the $\mathrm{Cl}$ concentration of that used by Long et al. (1996). It should be noted that this lower concentration of $\mathrm{Cl}$ has been used successfully by other investigators (Garde et al. 1997); further, the incidence of acrosome reaction in this study increased over time, indicating that this concentration is sufficient to induce the acrosome reaction in capacitated sperm.

In comparing the efficacy of inducing the acrosome reaction with heterologous, solubilised ZP, it was somewhat surprising to discover that ZP of the cat were more effective than those of the panda's closer relative, the black bear. However, this result may simply be more a reflection of oocyte quality than a species effect. For example, whereas the cat oocytes were harvested within only $2-5 \mathrm{~h}$ of ovarian excision, bear ovaries were frozen after excision, sometimes $12 \mathrm{~h}$ postmortem. A delay between death and harvest plus the tissue cryopreservation no doubt resulted in a higher incidence of oocyte degeneration in black bear than 
cat oocytes, perhaps compromising ZP composition. Additionally, cat oocytes underwent in vitro maturation prior to zonae harvesting, which may alter the acrosome reaction-inducing properties of the zonae. Regardless, it was important to recognise the efficacy of ZP from an evolutionarily distant family (i.e. the Felidae) inducing the acrosome reaction in a unique species in the family Ursidae. This finding substantiated earlier assertions for a lack of species specificity in the ability of the ZP to induce the acrosome reaction (Lee et al. 1987, Yoshimatsu et al. 1988, Slavik et al. 1990).

Giant panda sperm were able to capacitate and acrosome react in HEPES-buffered medium. Others have reported that HEPES is detrimental to capacitation (Visconti et al. 1999), but only if it contains no bicarbonate (Shi \& Roldan 1995). Our capacitation-supporting, HEPESbased medium contained bicarbonate that maintained the spermatozoa at the requisite $\mathrm{pH}$. This relatively simple finding is quite important when working in situations (as we were in this remote centre) where there was an absence of a $\mathrm{CO}_{2}$-regulated incubator. We expect that this finding will also be applicable when we eventually collect samples under even more rigorous conditions in the field.

Cryopreservation of giant panda spermatozoa did not alter the subsequent pace or overall incidence of capacitation compared with fresh counterparts until the final timepoint, and only in the HF10 treatment. This similarity among treatments most likely was an artifact of the increase in spontaneous acrosome reaction or damage in the control groups. Because we specifically defined a capacitated spermatozoon as one that was intact and capable of undergoing the acrosome reaction (by subtracting the percentage of sperm with intact acrosomes in treatment groups from control groups), the potential number of cells falling into this category decreased over time as more sperm were damaged or underwent acrosome reaction spontaneously. Nonetheless, the similarity of fresh and thawed sperm was surprising. In general, it is considered that the components of the egg yolk extenders used to protect the sperm during cryopreservation accelerate the capacitation process as determined by their ability to penetrate zona-free oocytes (Ijaz \& Hunter 1989, Ijaz et al. 1989, Bielfeld et al. 1990, Cormier et al. 1997), but detection of capacitation in sperm exposed to an egg yolk buffer using $\mathrm{Cl}$ may be suppressed compared with more physiological assays (Bielfeld et al. 1990). Bielfeld et al. (1990) found that $\mathrm{Cl}$ induced acrosome reaction in sperm that were incubated in a salt-based medium but not those exposed to egg yolk, whether the egg yolk was removed before testing or not (Bielfeld et al. 1990). So it is possible that a difference between the capacity of fresh and thawed sperm to capacitate existed but was masked by our assessment technique. However, if this was the case, we would have expected the ZP treatment to demonstrate any increased capacitation in thawed sperm.

Cryopreservation and thawing of sperm also appears to accelerate capacitation by inducing calcium flux and changes in lipid bilayer structure and tyrosine phosphorylation (Green \& Watson 2001). These spermatozoa often are capable of fertilisation and progressing into hyperactivity associated with capacitation (Eriksson et al. 2001); however, this suite of changes are of a destabilising nature and may contribute to reduced sperm longevity post-thawing (Samper 2001). Whether giant panda spermatozoa are less susceptible than other species to these perturbations (therefore explaining their ability to stay motile in vitro for long periods (Moore et al. 1984)) remains in question and requires further study. A logical priority would be to determine fresh versus frozen-thawed sperm longevity in vitro and in vivo. Thus, fresh and frozen giant panda sperm appear to spontaneously capacitate to an equivalent extent. It is possible that simply removing seminal plasma was sufficient to elicit membrane-destabilising effects similar to capacitation in the giant panda as it is in boar sperm (Maxwell \& Johnson 1999).

In summary, giant panda spermatozoa are readily induced to capacitate in vitro, and the acrosome reaction can be elicited using $\mathrm{Cl}$ or heterologous (felid or ursid) ZP. Most importantly, there is no detrimental effect on this requisite step to fertilisation using spermatozoa subjected to a rapid rate cryopreservation and thawing protocol. These results provide new fundamental information on a high profile, endangered species while suggesting that the fertility of cryopreserved-thawed giant panda spermatozoa will be similar to that following the use of fresh spermatozoa. This is critically important, as Al with fresh sperm has resulted in the birth of more than six cubs in the past 2 years alone (Xie Zhong, personal communication). The data presented here will likely increase the confidence of giant panda managers in testing frozenthawed sperm to achieve genetic management goals (Wildt et al. 2002).

\section{Acknowledgements}

We thank Lena May Bush for technical assistance, Dr Budhan Pukazhenthi for valuable advice, Jonathan Aaltonen and Abby Mathewson for technical assistance. Financial support was provided by Friends of the National Zoo, Morris Animal Foundation and the Smithsonian Institution's Women's Committee. The China Wildlife Conservation Association is a cooperative partner in the Smithsonian's National Zoological Park's research and conservation programme for giant pandas.

\section{References}

Andrews JC, Howard JG, Bavister BD \& Wildt DE 1992 Sperm capacitation in the domestic cat (Felis catus) and leopard cat (Felis bengalensis) as studied with a salt-stored zona pellucida penetration assay. Molecular Reproduction and Development 31 200-207.

Bielfeld P, Jeyendran R, Holmgren W \& Zaneveld L 1990 Effect of egg yolk medium on the acrosome reaction of human spermatozoa. Journal of Andrology 11 260-269.

Box G, Hunter J \& Hunter W 1978 Statistics for Experimenters: An Introduction to Design, Data Analysis, and Model Building. New York: John Wiley and Sons, Inc. 
Burgess C, Bredl J, Plummer J \& England G 2001 Vital and ultrastructural changes in dog spermatozoa during cryopreservation. Journal of Reproduction and Fertility Supplement 57 357-363.

Byrd W 1981 In vitro capacitation and the chemically induced acrosome reaction in bovine spermatozoa. Journal of Experimental Zoology 215 35-46.

Chen D, Ye Z \& Zhang Z 1984 Ultrastructure of giant panda spermatozoa. Acta Zoologica Sinica 30 301-304.

Chen D, Song X, Zhou X \& Duan C 1989a Study on in vitro sperm capacitation and egg penetration of giant panda. Science in China 32 435-441.

Chen D, Shi Q, Zhao X, Duan C, He G, Zhang A \& Feng W 1989b Studies of heterofertilization in vitro between giant panda and golden hamster. Acta Zoologica Sinica 35 276-380.

Chen M, Zhang G \& Mainke S 1994 Semen evaluation of giant pandas (Ailuropoda melanoleuca) at the Wolong Reserve. Zoo Biology $1383-86$

Cormier N, Sirard M \& Bailey J 1997 Premature capacitation of bovine spermatozoa is initiated by cryopreservation. Journal of Andrology 18 461-468.

Cummins J \& Yanagimachi R 1986 Development of ability to penetrate the cumulus oophorus by hamster spermatozoa capacitated in vitro in relation to the timing of the acrosome reaction. Gamete Research 15 187-212.

Dorval V, Dufour M \& Leclerc P 2002 Regulation of the phosphotyrosine content of human sperm proteins by intracellular $\mathrm{Ca}^{2+}$ : role of $\mathrm{Ca}^{2+}$-adenosine triphosphatases. Biology of Reproduction $\mathbf{6 7}$ $1538-1545$.

Drobnis E, Yudin A, Cherr G \& Katz D 1988 Hamster sperm penetration of the zona pellucida: kinematic analysis and mechanical implications. Developmental Biology 130 311-323.

Eriksson B, Vazquez J, Martinez E, Roca J, Lucas X \& Rodriguez-Martinez H 2001 Effects of holding time during cooling and of type of package on plasma membrane integrity, motility and in vitro oocyte penetration ability of frozen-thawed boar spermatozoa. Theriogenology 55 1593-1605.

Garde J, Ortiz N, Garcia A \& Gallego L 1997 Use of a triple-stain technique to detect viability and acrosome reaction in deer spermatozoa. Archives of Andrology 39 1-9.

Gil J, Rodriguez-Irazoqui M, Soderquist L \& Rodriguez-Martinez H 2002 Influence of centrifugation or low extension rates prefreezing on the fertility of ram semen after cervical insemination. Theriogenology 57 1781-1792.

Gillan L \& Maxwell W 1999 The functional integrity and fate of cryopreserved ram spermatozoa in the female tract. Journal of Reproduction and Fertility Supplement 54 271-283.

Gillan L, Evans G \& Maxwell W 1997 Capacitation status and fertility of fresh and frozen-thawed ram spermatozoa. Reproduction, Fertility and Development 9 481-487.

Green C \& Watson P 2001 Comparison of the capacitation-like state of cooled boar spermatozoa with true capacitation. Reproduction 122 889-898.

Harnal V, Spindler R, Monfort S, Pukazhenthi B, Bird D \& Wildt D 2001 Sperm capacitation in vitro in the Eld's deer. Theriogenology $56399-413$.

Harnal V, Wildt D, Bird D, Monfort S \& Ballou J 2002 Computer simulations to determine the efficacy of different genome resource banking strategies for maintaining genetic diversity. Cryobiology $44122-131$

Hodges J, Bevan D, Celma M, Hearn J, Jones D, Kleiman D, Knight J \& Moore H 1984 Aspects of the reproductive endocrinology of the female giant panda (Ailuropoda melanoleuca) in captivity with specific reference to the detection of ovulation and pregnancy. Journal of Zoology 203 253-267.

Howard JG 1993 Semen collection and analysis in non-domestic carnivores. In Zoo and Wild Animal Medicine III, pp 390-399. Ed. M Fowler. Philadelphia: WB Omaha, NE: Henry Doorly Zoo. Saunders Co.
Howard J 1999 Assisted reproductive techniques in nondomestic carnivores. In Zoo and Wild Animal Medicine: Current Therapy IV, pp 449-457. Eds M Fowler \& R Miller. Philadelphia: WB Saunders Co.

Howard JG, Brown JL, Bush M \& Wildt DE 1990 Teratospermic and normospermic domestic cats: ejaculate traits, pituitary-gonadal hormones and improvement of spermatozoal motility and morphology after swim-up processing. Journal of Andrology 11 204-215.

Hu J \& Wei F 1990 Development and progress of breeding and rearing giant pandas in captivity within China. In Research and Progress in Biology of the Giant Panda, pp 322-325. Eds J Hu, F Wei, C Yuan \& T Wu. Sichuan: Sichuan Publishing.

Huang Y, Li DS, Zhang H, Du J, Zhang G, Wei RP, McGeehan L, Howard JG, Tang C \& Wang PY 2001 Artificial insemination of a giant panda with cooled semen: an effort to promote genetic diversity in a captive population. Chinese Journal of Applied Environmental Biology 7 558-562.

Huang Y, Wang PY, Zhang G, Zhang H, Li DS, Du J, Wei RP, Tang C, Spindler RE, Wildt DE \& Howard JG 2002 Use of artificial insemination to enhance propagation of giant pandas at the Wolong breeding center. Proceedings of the 2nd International Symposium on Assisted Reproductive Technology (ART) for the Conservation and Genetic Management of Wildlife, pp 172-175. Omaha, NE: Henry Doorly Zoo.

Hunter R \& Hall J 1974 Capacitation of boar spermatozoa: synergism between uterine and tubal environments. Journal of Experimental Zoology 188 203-214.

ljaz A \& Hunter A 1989 Induction of bovine sperm capacitation by TEST-yolk semen extender. Journal of Dairy Science $\mathbf{7 2}$ 2683-2690.

ljaz A, Hunter A \& Graham E 1989 Identification of the capacitating agent for bovine sperm in egg yolk-TEST semen extender. Journal of Dairy Science 72 2700-2706.

Johnston LA, O'Brien SJ \& Wildt DE 1989 In vitro maturation and fertilization of domestic cat follicular oocytes. Gamete Research $\mathbf{2 4}$ $343-356$.

Johnston LA, Donoghue AM, Igo W, Simmons LG, Wildt DE \& Rieffenberger J 1994 Oocyte recovery and maturation in the American black bear (Ursus americanus): a model for endangered ursids. Journal of Experimental Zoology 269 53-61.

Kopf G \& Gerton G 1991 The mammalian sperm acrosome and the acrosome reaction. In Elements of Mammalian Fertilization, pp 153-203. Ed. P Wasserman. Boca Raton, Florida: CRC Press.

Leclerc P, Lamirande Ed \& Gagnon C 1996 Cyclic adenosine $3^{\prime}, 5^{\prime}$ monophosphate-dependent regulation of protein tyrosine phosphorylation in relation to human sperm capacitation and motility. Biology of Reproduction 55 684-692.

Lee M, Trucco G, Bechtol K, Wummer N, Kopf G, Blasco L \& Storey B 1987 Capacitation and acrosome reaction in human spermatozoa monitored by chlorotetracycline fluorescence assay. Fertility and Sterility 48 649-658.

Lefievre L, De Lamirande E \& Gagnon C 2000 The cyclic GMPspecific phosphodiesterase inhibitor, sildenafil, stimulates human sperm motility and capacitation but not acrosome reaction. Journal of Andrology 21 929-937.

Liu D, Fang J, Sun R, Zhang G, Wei R \& Zhang H 1998 Behavioural comparison in individuals of different sexual ability in giant panda (Ailuropoda melanoleuca). Acta Zoologica Sinica 44 27-34.

Liu W 1981 A note on the artificial insemination of giant panda. Acta Zoologica Sinica 12 73-76.

Liu W, Ye J, Li C \& Liao G 1979 Artificial insemination experiment on the giant panda. The Chinese Zoo Annual 2 20-24.

Long JA, Wildt DE, Wolfe BA, Critser JK, DeRossi RV \& Howard JG 1996 Sperm capacitation and the acrosome reaction are compromised in teratospermic domestic cats. Biology of Reproduction $\mathbf{5 4}$ 638-646.

Lu Z, Johnson W, Menotti-Raymond M, Yuhki N, Martenson J, Mainka S, Shiqiang H, Qingguo Z, Zhihe Z, Li G, Pan W \& 
O'Brien S 2001 Patterns of genetic variation in remaining giant panda (Ailuropoda melanoleuca) populations. Conservation Biology 5 1596-1607.

Masui M, Hiramatsu H, Nose N, Nakazato R, Sagawa Y, Tajima H \& Saito K 1989 Successful artificial insemination in the giant panda (Ailuropoda melanoleuca) at Ueno Zoo. Zoo Biology 8 17-26.

Maxwell W \& Johnson L 1999 Physiology of spermatozoa at high dilution rates: the influence of seminal plasma. Theriogenology $\mathbf{5 2}$ 1353-1362.

Miller RG 1981 Simultaneous Statistical Inference. New York: Springer-Verlag

Moore H, Bush M, Celma M, Garcia A, Hartman T, Hearn J, Hodges J, Jones D, Knight J, Monsalve L \& Wildt D 1984 Artificial insemination in the giant panda (Ailuropoda melanoleuca). Journal of Zoology 203 269-278.

O'Brien J \& Roth T 2000 Functional capacity and fertilizing longevity of frozen-thawed scimitar-horned oryx (Oryx dammah) spermatozoa in a heterologous in vitro fertilization system. Reproduction, Fertility and Development 12 413-421.

Parrish JJ, Susko-Parrish J, Winer MA \& First NL 1988 Capacitation of bovine sperm by heparin. Biology of Reproduction $\mathbf{3 8}$ 1171-1180.

Parrish J, Susko-Parrish J \& First N 1989 Capacitation of bovine sperm by heparin: inhibitory effect of glucose and role of intracellular pH. Biology of Reproduction 41 683-699.

Perreault S 1990 Regulation of sperm nuclear reactivation during fertilization. In Fertilization in Mammals, pp 285-296. Eds B Bavister, J Cummins \& E Roldan. Norwell: Serono Symposia.

Platz CC, Wildt DE, Howard JG \& Bush M 1983 Electroejaculation and semen analysis and freezing in the giant panda (Ailuropoda melanoleuca). Journal of Reproduction and Fertility 67 9-12.

Pope C, Zhang Y \& Dresser B 1991 A simple staining method for evaluating acrosomal status of cat spermatozoa. Journal of Zoo and Wildlife Medicine 22 87-95.

Pukazhenthi B, Long J, Wildt D, Bush M \& Howard J 1996 Capacitation of clouded leopard sperm: II. Effect of calcium and cyclic AMP on acrosome reaction and zona penetration. Journal of Andrology 46 (Suppl 94).

Pukazhenthi BS, Wildt DE, Ottinger MA \& Howard JG 1998 Inhibition of domestic cat spermatozoa acrosome reaction and zona pellucida penetration by tyrosine kinase inhibitors. Molecular Reproduction and Development 49 48-57.

Rodriguez-Martinez H, Larsson B \& Pertoft H 1997 Evaluation of sperm damage and techniques for sperm clean-up. Reproduction, Fertility and Development 9 297-308.

Samper J 2001 Management and fertility of mares bred with frozen semen. Animal Reproduction Science 68 219-228.

Schaller G, Hu J, Pan W \& Zhu J 1985 The Giant Pandas of Wolong. Ed. G Schaller. Chicago, IL: University of Chicago Press.

Shi Q-X \& Roldan ERS 1995 Bicarbonate/ $\mathrm{CO}_{2}$ is not required for zona pellucida- or progesterone-induced acrosomal exocytosis of mouse spermatozoa, but is essential for capacitation. Biology of Reproduction 52 540-546.

Slavik T, Pavlok A \& Fulka J 1990 Penetration of intact bovine ova with ram sperm. Molecular Reproduction and Development 25 345-347.

Spindler RE, Howard JG, Huang Y, Li DS, Zhang H \& Wildt DE 2001 Cryopreservation has no impact on subsequent sperm capacitation in vitro in the giant panda. Biology of Reproduction 64 (Suppl) 522.

Suarez S, Katz D \& Meizel S 1984 Changes in motility that accompany the acrosome reaction in hyperactivated hamster spermatozoa. Gamete Research $10253-265$.
Sun Q, Liu H, Li X, Song X, Yu J, Li G \& Chen D 1996 The role of $\mathrm{Ca}^{2+}$ and protein kinase in the acrosome reaction of giant panda (Ailuropoda melanoleuca) spermatozoa. Theriogenology $\mathbf{4 6}$ 359-367.

Talbot P 1985 Sperm penetration through oocyte investments in mammals. American Journal of Anatomy 174 331-346.

Thundathil J, de Lamirande E \& Gagnon C 2002 Different signal transduction pathways are involved during human sperm capacitation induced by biological and pharmacological agents. Molecular Human Reproduction 8 811-816.

United-States-Fish-and-Wildlife-Service 1998 Giant Panda Import Permit Policy 45839-45854 Federal Register Doc. 98-23074.

Uto N, Yoshimatsu N \& Lopata A 1988 The zona-induced acrosome reaction of hamster spermatozoa. Journal of Experimental Zoology $248113-120$.

Visconti P, Stewart-Savage L, Blasco A, Battaglia L, Miranda P, Kopf G \& Tezon J 1999 Roles of bicarbonate, cAMP and protein tyrosine phosphorylation on capacitation and the spontaneous acrosome reaction of hamster sperm. Biology of Reproduction 61 76-84.

Wildt DE, Rall WF, Critser JK, Monfort SL \& Seal US 1997 Genome resource banks: living collections for biodiversity conservation. Bioscience 47 689-698.

Wildt DE, Ballou J, Miller P, Traylor-Holzer K \& David V 2002 Report of Genetic Management for Giant Pandas Ex Situ Workshop. Eds DE Wildt \& J Ballou. Smithsonian National Zoological Park, Chinese Association of Zoological Gardens and China Wildlife Conservation Association Chengdu, China.

Wolfe BA \& Wildt DE 1996 Development to blastocysts of domestic cat oocytes matured and fertilized in vitro after prolonged cold storage. Journal of Reproduction and Fertility 106 135-141.

Yan X, Deng X, Zhang H, Lam M, Ellis S, Wildt D, Miller P \& Seal U 2000 Giant Panda Conservation. Assessment and Research Techniques Workshop Draft Report. Eds DE Wildt \& S Ellis. Conservation Breeding Specialist Group IUCN/SSC, Apple Valley, Chengdu, China.

Yanagimachi R 1994 Mammalian Fertilization. In The Physiology of Reproduction, pp 189-317. Eds. E Knobil \& J Neil. New York: Raven Press.

Ye Z, He G, Zhang A, Song Y, Xu Q \& Feng W 1991 Studies on the artificial pollination method of giant panda. Journal of Sichuan University (Nature Science) 28 50-53.

Yoshimatsu N, Yanagimachi R \& Lopata A 1988 Zonae pellucidae of salt-stored hamster and human eggs: their penetrability by homologous and heterologous spermatozoa. Gamete Research 21 $115-126$.

Zhang A, Ye Z, He G, Xu Q \& Song Y 1991 Studies on conception effect of the frozen semen in the giant panda: a study on breeding and disease of the giant panda. In Pandas Special Issue, pp 91-97. Ed. A Zhang. Sichuan: Sichuan Scientific and Technologic Publishing House.

Zhang G, Swaisgood R \& Zhang H 2004 An evaluation of behavioral factors influencing reproductive success and failure in captive giant pandas. International Journal of Comparative Psychology (In Press).

Zheng S, Zhao Q, Xie Z, Wildt D \& Seal US 1997 Report of Giant Panda Captive Management Planning Workshop, Conservation Breeding Specialist Group IUCN/SSC, Apple Valley, Chengdu, China.

Received 7 October 2003

First decision 7 January 2004

Accepted 2 February 2004 